\title{
Photonic crystal fiber micro pressure sensor based on an extrinsic Fabry-Perot interferometer fabricated by femtosecond laser
}

\author{
Peng Wang1, a, Liuchao Zhang2, b, Feifei Chen2, c, and Zhitao Cao1, d \\ ${ }^{1}$ Laser Micro/Nano Fabrication Laboratory, School of Mechanical Engineering, Beijing Institute of \\ Technology, Beijing 100081, China \\ ${ }^{2}$ School of Optoelectronics, Beijing Institute of Technology, Beijing 100081, China \\ a845257907@qq.com, bzhangliuchao1991@163.com, ${ }^{c} 191632492 @ q q . c o m,{ }^{d}$ \\ zhitao_c@126.com, ${ }^{e}$ wangsumei@bit.edu.cn
}

\begin{abstract}
Keywords: Fabry-Perot; Fiber optics sensors; Microstructure fabrication; Pressure measurement Abstract. A photonic crystal fiber extrinsic Fabry-Perot interferometric (FPI) micro pressure sensor is fabricated by femtosecond laser (fs) and fusion splicing. The pressure sensitivity can be adjusted by controlling the thickness of the diaphragm. The laser mircomachined FPI cavity helps to reduce the temperature-pressure cross-sensitivity. A pressure sensitivity of $282 \mathrm{~nm} / \mathrm{MPa}$ in terms of cavity length change is demonstrated within $1 \mathrm{MPa}$.
\end{abstract}

\section{Introduction}

Fiber-optic pressure sensors have been applied in down-hole pressure monitoring [1], engines [2], aerodynamics [3], biomedical field [4], etc. In recent years, fiber-optic extrinsic FPI pressure sensors based on diaphragm deformation have been studied. The diaphragm is deformed when the pressure is changed. The cavity length is changed, the interference spectrum will be shifted, pressure is measured. Some methods have been put forward by direct creation of a micro FPI cavity on an optical fiber, such as laser micromaching [5- 6], selective etching of the fiber core [7], and lithography [8] etc. A fs laser fabricated FPI fiber tip pressure sensor is immune to variations in the environment refractive index. [5] The whole sensor head is etched, so that the mechanical strength is weakened. [7] The diaphragms are attached to seal the microcavity, including chemical vapor deposition of grapheme [9], bonding of a polymeric diaphragm [10] and attachment of silk fibroin. [11] The grapheme-diaphragm based sensor had a problem of leakage. [9] The polymeric diaphragm was fabricated by a three-layer diaphragm design: PDMS, parylene, and gold. Upon heating, the thermal mismatch between three-layer diaphragm usually results in the fracture of the sensor. [10] The silk fibroin diaphragm based sensor shows a response of $12.3 \mathrm{~nm} / \mathrm{kPa}$ in terms of cavity length change, but the measured maximum pressure is only $80 \mathrm{kPa}$. It is limited by the mechanical strength of the silk fibroin. [11]

Fs laser micromachining has the advantages of non-heat affected zone, ultra high precision, free from limitation of various materials and bulk machining. Fs laser has been a useful tool to machine micro structures in fiber such as a micro trench in the fiber taper [12], a semi-circular structure of the gold film coated on the fiber end face [13] and optofluidic microchannels [14].

In this paper, a micro extrinsic FPI pressure sensor is fabricated and tested. The sensor was made by fs laser micromachining and fusion splicing. A silica diaphragm was used to seal the microcavity. The size of the sensor head is within tens of micrometers, and the temperature-pressure cross-sensitivity of the sensor is low.

\section{Sensor structure and principle}

The structure of the sensor is illustrated in Fig. 1 (a). The fabrication procedure is described in detail. The endless single mode PCF was firstly collapsed by using arc discharging. Secondly, a micro hole was fabricated at the end face of the PCF by fs laser micromachining. Thirdly, the hole-drilled PCF was spliced to a segment of the silica rod. During splicing the hole surface was melted to get smooth by the arc. A sealed air cavity was formed in between the PCF and the silica rod. Then, the silica rod was 
precisely cleaved at the position of about fifty micrometers away from the splicing point. Finally the fs laser micromachining was applied to reduce the thickness of the silica rod so that a thin piece of silica rod was left to perform as the diaphragm. The external diaphragm surface is rough and uneven.

(a)
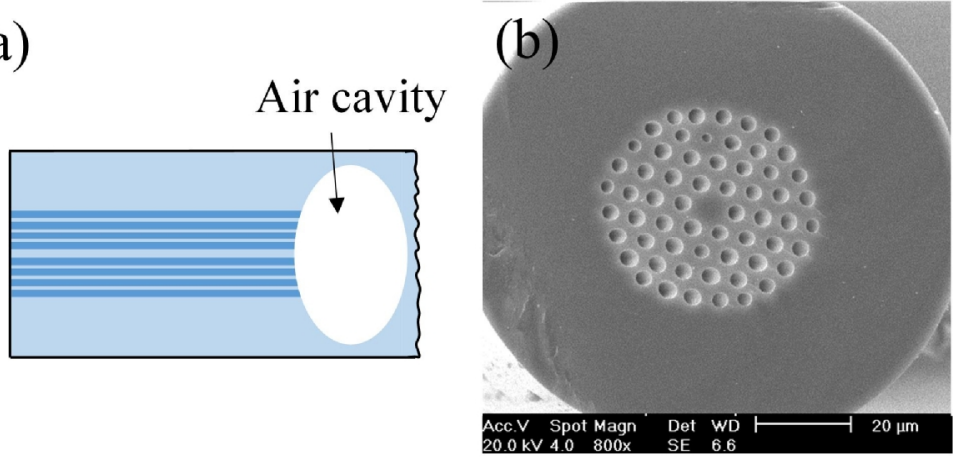

(c)

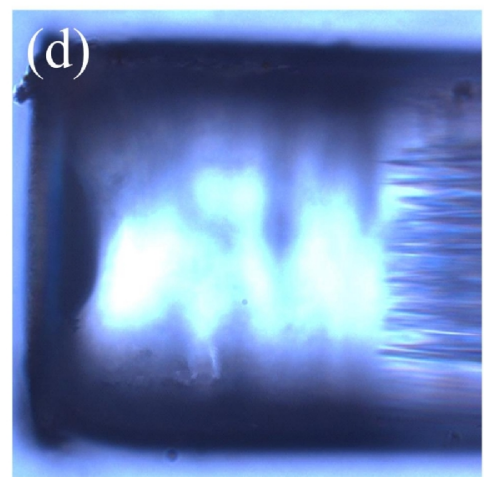

Fig. 1 (a) Structure of the sensor head. (b) SEM image of the end face of the PCF. (c) Microscope image of the PCF collapsed region. (d) Microscope image of the sensor head

A segment of the PCF is served as the lead in fiber. The sensor head will generate two reflections, one from the cavity bottom and the other from the inner surface of the diaphragm. The two reflected light arrives in a phase change of $2 L / \lambda$ at the bottom of the micro hole, yielding interference, where $L$ is the cavity length, $\lambda$ is the wavelength. If the external environment pressure changes, the diaphragm will deform its shape, and the interference spectrum will change accordingly, allowing the calculation of the phase shift caused by the pressure variation. The pressure sensitivity is defined as the diaphragm deflection over the applied pressure. The pressure sensitivity is [15]

$$
S=\frac{3\left(1-\mu^{2}\right) a^{4}}{16 E h^{3}}(\mu \mathrm{m} / \mathrm{Pa})
$$

where $a$ and $h$ are the radius and thickness of the diaphragm(in $\mu \mathrm{m}), E=73 \mathrm{GPa}$ is the Young's modulus and $\mu=0.17$ is the Poisson' ratio of the fused silica.

The relationship between the change of the cavity length and the wavelength shift of the interference valley is given by

$$
\frac{\Delta L}{2 L}=\frac{\Delta \lambda_{v}}{\lambda_{v}} .
$$

where $\lambda_{v}$ is the wavelength of a specific interference valley, $\Delta \lambda_{v}$ is the change in the center wavelength of that specific interference valley, $\Delta L$ is the change of the cavity length, and $L$ is the initial cavity length. The pressure sensitivity of the fabricated sensor was characterized.

\section{Sensor fabrication and pressure test}

The microscope image of the PCF end face is shown in Fig. 1(b). The diameter of the core is $13 \pm 0.3 \mu \mathrm{m}$, while the diameter of the cladding is $125 \pm 5 \mu \mathrm{m}$. There are four rings of air holes 
surrounding the core. The mode field diameter of the PCF at $1550 \mathrm{~nm}$ is $14.3 \mu \mathrm{m}$ which is larger than that of the single mode fiber.

The experimental setup is shown in Fig. 2. A homemade interrogator [16] is used to acquire the interference spectrum. The sensor head was sealed into a stainless steel chamber. An air pump was connected to the chamber to pump the compressed air. A pressure meter was used to monitor the air pressure in the chamber.

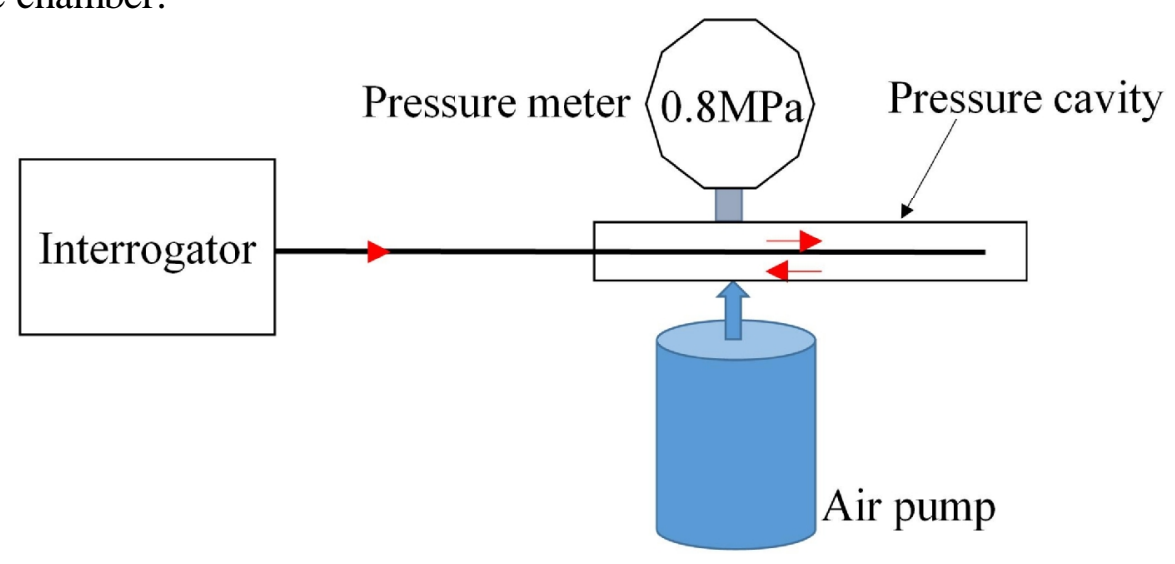

Fig. 2 Experimental setup of the pressure test

The end of the PCF was firstly collapsed by using arc discharging. The arc current and time were set as $30 \%$ and $400 \mathrm{~ms}$, respectively. The length of the collapsed region is measured to be about $87.48 \mu \mathrm{m}$, as shown in Fig. 1(c). With the air holes collapsed, the bottom surface fabricated by fs laser is flatter than the untreated PCF. What's more, the acetone can be prevented from entering into the air holes during an ultrasonic bath after femtosecond laser fabricaiton.

The fs laser(Spectra-Physics, Inc.) with $800 \mathrm{~nm}$ center wavelength, $35 \mathrm{fs}$ pulse width and $1 \mathrm{kHz}$ repetition rate was attenuated by a combination of a half wave plate and a polarizer and focused by an objective lens(Olympus LMPlanFL N 50x with a N.A. of 0.50). The laser energy on target was attenuated to be $0.15 \mu \mathrm{J}$ per pulse. The fiber was positioned upright on a six degree of freedom motion stage. Helical drilling was used to drilling a micro hole with the diameter of $80 \mu \mathrm{m}$ and the depth of $20 \mu \mathrm{m}$ at the end face of the PCF. During drilling, the plasma blew away by the compressed air. When the fabrication was completed, the PCF was cleaned by an ultrasonic bath with acetone for 5 minutes. The PCF was fused together with a silica rod with a diameter of $125 \mu \mathrm{m}$. The same arc current and time were used to avoid collapsing of the micro hole.

The silica rod was cleaved under a microscope(magnification 160x). The typical length of the remained silica rod is around $50 \mu \mathrm{m}$. The sensor had a distorted spectrum. The fs laser machined surface is rougher than the cleaved surface. Hence, the reflectivity of the bottom surface is lower than the inner and outer surfaces of the diaphragm. 


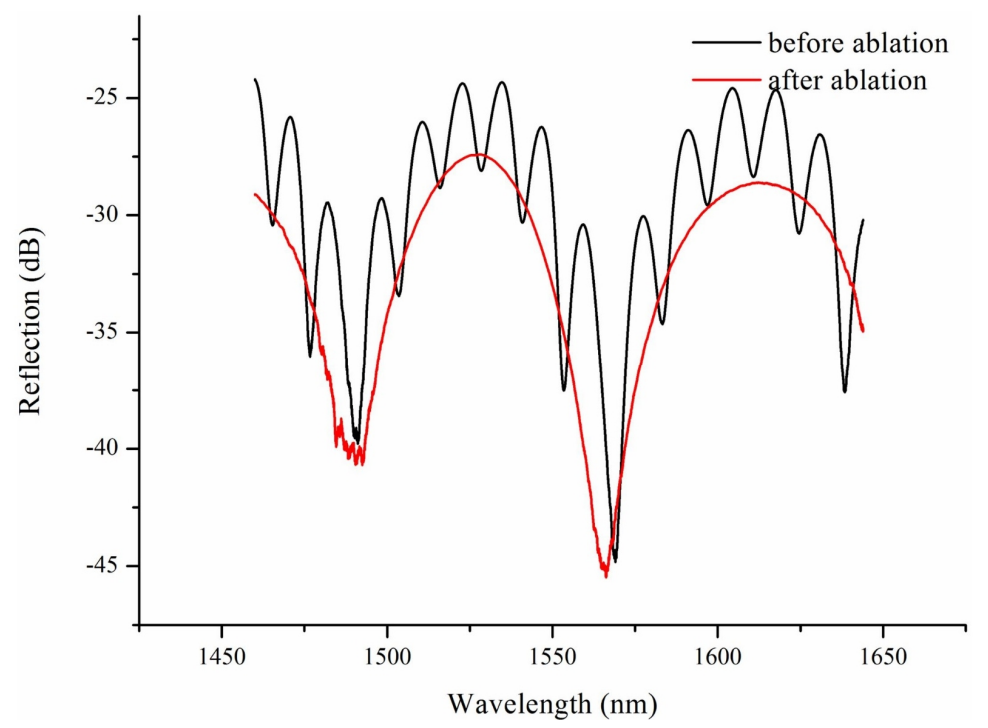

Fig. 3 Reflection spectra of the sensor, the black line represents the distorted interference spectrum and the red line is the two beam interference spectrum after fs laser ablation

Then the silica rod was etched by fs laser to a thickness of several micrometers. The silica rod was removed layer by layer. After fs laser micromachining, the reflection from the outer surface is eliminated. The interference pattern was a two beam interference, as shown in Fig. 3. According to the equation,

$$
L=\frac{\lambda_{1} \cdot \lambda_{2}}{2000 \cdot n_{\text {silica }} \cdot\left(\lambda_{1}-\lambda_{2}\right)}(\mu \mathrm{m})
$$

the cavity length is calculated to be $15 \mu \mathrm{m}$. The microphotograph is shown in Fig. 1 (d).

The sensor head was sealed into a stainless steel pressure chamber. Turned on the pump to inject the compressed air into the chamber. When the pressure measured by the pressure meter reached at $1.1 \mathrm{MPa}$, turned the air pump off. As the pressure decreased gradually, the interference spectra were recorded from $1 \mathrm{MPa}$ to $0 \mathrm{MPa}$ at a step of $0.1 \mathrm{MPa}$.

\section{Results and discussion}

The interference spectra at different pressures are analyzed by using spline interpolation and curve fitting, as shown in Fig. 4. As the pressure increases, the interference spectrum shifts to the short wavelength direction. The diaphragm bents inward and the cavity length decreases. 


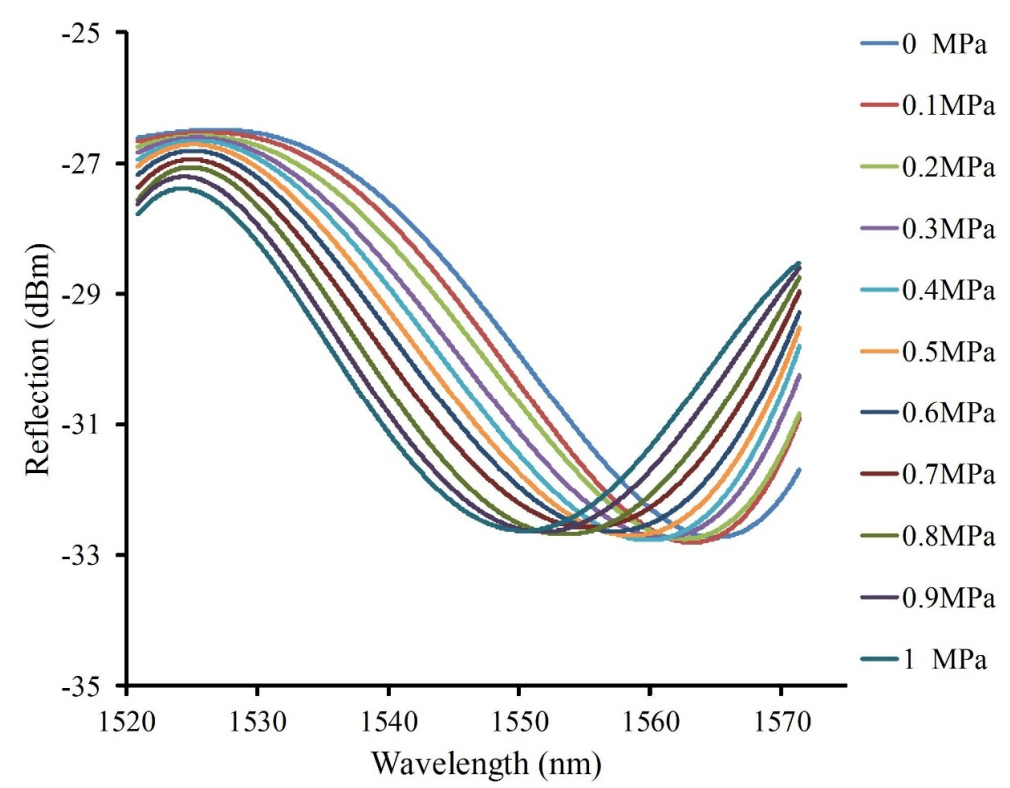

Fig. 4 Interference spectra of the FPI sensor at different pressures

Fig. 5 shows the results obtained from the sensor, where the changes in cavity length are plotted as a function of the applied pressure. Based on the linear fitting curve, the sensitivity of the sensor is calculated to be $282 \mathrm{~nm} / \mathrm{MPa}$, which can be further improved by decreasing the thickness of the diaphragm. The diaphragm thickness could be calculated from measured sensitivity using Eq. (1), which is about $2.8 \mu \mathrm{m}$.

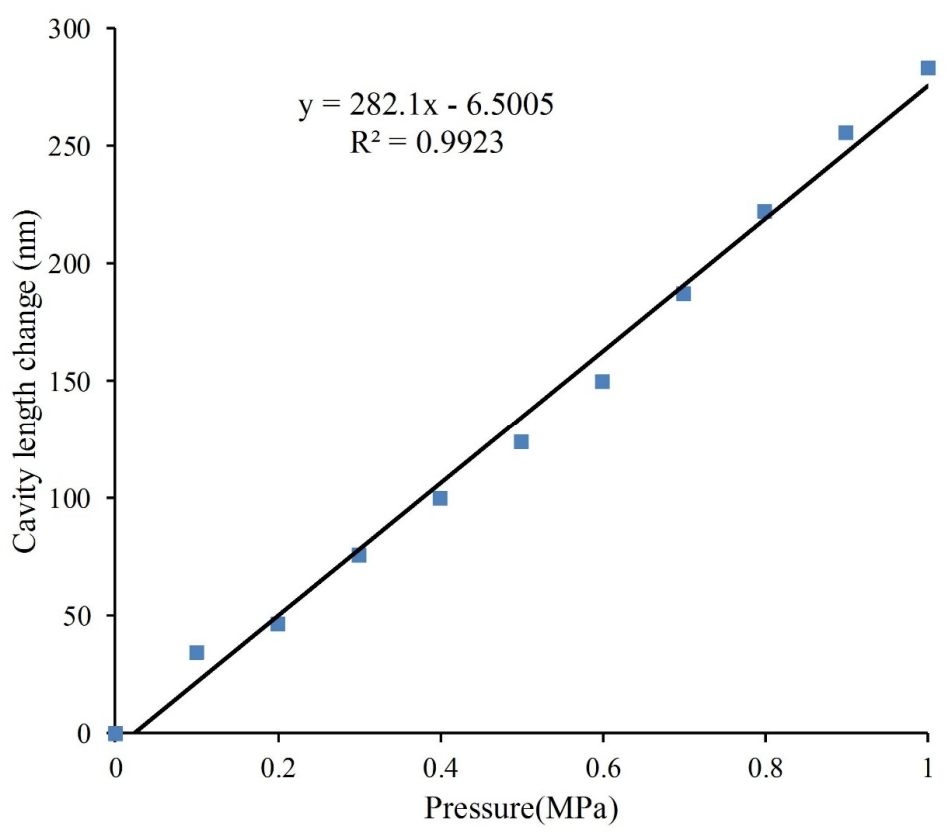

Fig. 5 Pressure-induced interferogram shift of FPI sensor.

\section{Conclusion}

A fiber optic pressure sensor based on Fabry-Perot interferometer is fabricated by fs laser micromachining and fusion splicing. The sensor head is made of PCF and silica rod. The sensor's size is small than the dimensions of optical fiber. The microcavity has a length of $15 \mu \mathrm{m}$. The diaphragm is machined and etched by fs laser to a thickness of about $2.8 \mu \mathrm{m}$. The pressure sensitivity is $282 \mathrm{~nm} / \mathrm{MPa}$ within $1 \mathrm{MPa}$ and can be further improved by decreasing the thickness of the diaphragm. The sensor has a low temperature pressure cross-sensitivity since the air cavity is not sensitive to temperature 
variation. The all-fused-silica structure makes the sensor has potential applications for pressure measurement in a high temperature environment.

\section{Acknowledgements}

This work was supported by the 863 Program of the Ministry of Science and Technology of China [No. 2015AA043504]

\section{References}

[1] S. H. Aref, M. I. Zibaii and H. Latifi: Meas. Sci. Technol. Vol. 20 (2009), p. 034009

[2] T. Bae, R.A. Atkins, H. F. Taylor, and W. N. Gibler: Appl. Opt. Vol. 42 (2003), p. 1003

[3] N. J. Lawson, R. Correia , S. W. James, M. Partridge, S. E. Staines, J. E. Gautrey, K. P. Garry, J. C. Holt and R. P. Tatam: Meas. Sci. Technol. Vol. 27 (2016), p. 104001

[4] S. Poeggel, D. Duraibabu, K. Kalli, G. Leen, G. Dooly, E. Lewis, J. Kelly and M. Munroe: Biosensors Vol. 5 (2015), p. 432

[5] Y.N. Zhang, L. Yuan, X.W. Lan, A. Kaur, J. Huang, and H. Xiao: Opt. Lett. Vol. 38 (2013), p. 4609

[6] Z.L. Ran, S. Liu, Q. Liu, Y.J. Wang, H.H. Bao, and Y.J. Rao: IEEE Sens. J. Vol. 15 (2015), p. 3955

[7] Y.Z. Zhu, and A.B. Wang: IEEE Photonic Tech. L. Vol. 17 (2005), p.447

[8] J.L. Zhu, M. Wang, L. Chen, X.Q. Ni, H.B. Ni: Opt. Fiber Technol. Vol. 34 (2017), p. 42

[9] J. Ma, W. Jin, H. L. Ho, and J. Y. Dai, Opt. Lett. Vol. 37 (2012), p. 13

[10] J. Eom, C.J. Park, B. H. Lee, J.H. Lee, I. B. Kwon, E. Chung: Sen. and Act. A 225 (2015), p. 25

[11] L. Cheng, C. Wang, Y. Huang, H. Liang, B.O. Guan: Opt. Express Vol. 24 (2016), p.19600

[12] Z.T. Cao, L. Jiang, S.M. Wang, P. Wang, F. Zhang, and Y.F. Lu: Appl. Opt. 53(2014), p. 1028

[13] P.F. Liu, L. Jiang, S.M. Wang, Z.T. Cao, and P. Wang: Chin. Opt. Lett. Vol. 14 (2016), p. 020602

[14] L. Yuan, J. Huang, X.W. Lan, H.Z. Wang, L. Jiang, and H. Xiao: Opt. Lett. Vol. 39 (2014), 2358

[15] M. D. Giovanni: Flat and Corrugated Diaphragm Design Handbook (M. Dekker, 1982).

[16] Y. Jiang: IEEE Photonics Technol. Lett. Vol. 20 (2008), p. 75 